\title{
CALCULATING THE NEAR FIELD OF A LINE OF SOURCES USING MELLIN TRANSFORMS
}

\author{
P. A. MARTIN' 1
}

(Received 29 August 2006; revised 13 July 2007)

\begin{abstract}
In slender-body theories, one c ften has to find asymptotic approximations for certain integrals, representing distribution: of sources along a line segment. Here, such approximations are obtained by a systematic riethod that uses Mellin transforms. Results are given near the line (using cylindrical polar coordinates) and near the ends of the line segment (using spherical polar coordinates).
\end{abstract}

2000 Mathematics subject cla: sification: primary 41 A60; 44A1S; secondary $31 \mathrm{~B} 10$.

Keywords and phrases: Mellis transforms; asymptotic approximations; slender-body theory.

\section{Introduction}

Slender-body theory is a clas: ic topic in fluid dynamics; see, for example, [6, Section 11.2]. A typical (and sin ple) problem is as follows. Consider a rigid, axisymmetric body, defined by $r=i(x), 0 \leq x \leq L$, where $r$ and $x$ are cylindrical polar coordinates, $L$ is the length of the body and $S(x)$ is a given function defining the shape of the body. For the body to se slender, it is required that $S / L \ll 1$ for all $x$. We suppose that the body is in an ideal fluid, and that there is an incident axisymmetric flow. Then the perturbation to this flow caused by the presence of the body will also be axisymmetric, and so it is plausible that the velocity potential for the perturbation, $\phi(r, x)$, can be represented as a distribution of sources along a piece of the $x$-axis inside the body,

$$
\phi(r, x)=\int_{\alpha}^{\beta} \frac{m\left(x_{1}\right) d x_{1}}{\sqrt{\left(x-x_{1}\right)^{2}+r^{2}}}
$$

\footnotetext{
'Department of Mathematical and Computer Sciences, Colorado School of Mines, Golden, Colorado 80401-1887, USA; email: pmartin@mines.edu.

(C) Australian Mathematical Society 2007, Serial-fee code 1446-1811/07
} 
where $m$ is a density function and $0 \leq \alpha<\beta \leq L$. Slender-body theory gives methods for estimating $m, \alpha$ and $\beta$. There is a considerable literature on these methods, covering their derivation and application, and their generalization to other physical problems. We mention a few papers from the 1960 s $[8,9,12,16]$, Tuck's review [17], and the more recent work of Sellier [15], Chadwick [3] and Petrov [14].

A key ingredient in slender-body theory is the approximation of integrals such as (1.1) for small values of $r$; this is the subject of this short paper. Methods for approximating (1.1) are reviewed in Section 2. We then describe another method (in Section 3), using Mellin transforms. This method has several virtues: it is systematic, it is rigorous and it yields the complete asymptotic expansion. Further results are given in Section 4, with emphasis on the behaviour of $\phi$ near the end-points. In particular, we examine $\phi$ as a function of distance from the end-point at $x=\alpha$.

\section{A short review}

To begin with, suppose that $\alpha=-\infty$ and $\beta=\infty$. Then, Tuck [17] shows that

$$
\phi(r, x)=-2 m(x) \log r+f(x)+O\left(r^{2} \log r\right) \quad \text { as } r \rightarrow 0,
$$

where

$$
f(x)=\int_{-\infty}^{\infty} m^{\prime}(\xi) L(x-\xi) d \xi \quad \text { and } \quad L(x)= \begin{cases}\log (2 x), & x>0 \\ -\log (-2 x), & x<0\end{cases}
$$

Thus, "three dimensional [point] source distributions along a line look like two dimensional line sources when we are close to them" [17, page 185]. This accounts for the first term on the right-hand side of (2.1). The second term is surprisingly complicated, and reveals a non-local dependence on the density function $m$. Tuck describes two ways to derive (2.1), one involving Fourier transforms, the other involving a splitting of the range of integration.

Goldstein [6, pages 183-185] derives an approximation for finite $\alpha$ and $\beta$,

$$
\begin{aligned}
\phi(r, x) \simeq & -2 m(x) \log \left(\frac{r}{2}\right)+m(\alpha) \log (x-\alpha)+m(\beta) \log (\beta-x) \\
& +\int_{\alpha}^{x} m^{\prime}(\xi) \log (x-\xi) d \xi-\int_{x}^{\beta} m^{\prime}(\xi) \log (\xi-x) d \xi,
\end{aligned}
$$

for $\alpha<x<\beta$. (For $x=\alpha$, see (4.5).) Equation (2.3) can be written as (2.1) (without the error term) with

$$
f(x)=m(\alpha) \log 2(x-\alpha)+m(\beta) \log 2(\beta-x)+\int_{\alpha}^{\beta} m^{\prime}(\xi) L(x-\xi) d \xi .
$$


Sellier [15] has described a very general scheme for approximating integrals such as (1.1). The result is an expansion in which the terms are given as certain finite-part integrals: the main difficulty "consists in carefully calculating" these integrals [15, page 1748]. Sellier also states [15, page 1738] that integrals such as (1.1) cannot be approximated using Mellin-transform techniques: this is false, as we show in the next section.

\section{Use of Mellin transforms}

We want to approximate $\phi(r, x)$, and we assume for now that $\alpha<x<\beta$. We also assume that $m$ is sufficiently smooth. See Section 4 for other possibilities.

We start with some preliminary transformations. Suppose that, without loss of generality, $x$ is closer to $\alpha$ than $\beta$; put $x-\alpha=a$ and $\beta-x=b>a$. Then, after putting $x_{1}=x+\xi$ in (1.1), we obtain

$$
\phi(r, x)=\int_{-a}^{a} \frac{m(x+\xi)}{\sqrt{\xi^{2}+r^{2}}} d \xi+\int_{a}^{b} \frac{m(x+\xi)}{\sqrt{\xi^{2}+r^{2}}} d \xi=I_{1}(r, x)+I_{2}(r, x),
$$

say. The second integral can be expanded using the Maclaurin expansion

$$
\frac{1}{\sqrt{1+t^{2}}}=\sum_{n=0}^{\infty} \frac{\Gamma(n+1 / 2)}{\sqrt{\pi} n !}\left(-t^{2}\right)^{n}, \quad|t|<1 ;
$$

the result is

$$
I_{2}(r, x)=\sum_{n=0}^{\infty} c_{n}(x)\left(-r^{2}\right)^{n}
$$

with

$$
c_{n}(x)=\frac{\Gamma(n+1 / 2)}{\sqrt{\pi} n !} \int_{2 x-\alpha}^{\beta} \frac{m(t) d t}{(t-x)^{2 n+1}} .
$$

Next, consider the first integral in (3.1), $I_{1}$. The range of integration is symmetric about $\xi=0$, so we split $m$ into its even and odd parts. Thus, we write

$$
\begin{aligned}
m(x+\xi) & =m_{\mathrm{e}}(\xi ; x)+m_{\mathrm{o}}(\xi ; x), \quad \text { where } \\
m_{\mathrm{e}}(\xi ; x) & =\frac{m(x+\xi)+m(x-\xi)}{2} \quad \text { and } \quad m_{0}(\xi ; x)=\frac{m(x+\xi)-m(x-\xi)}{2}
\end{aligned}
$$

evidently, $m_{0}$ integrates to zero, giving

$$
I_{1}(r, x)=2 \int_{0}^{a} \frac{m_{\mathrm{e}}(\xi ; x)}{\sqrt{\xi^{2}+r^{2}}} d \xi
$$


Also, as $m_{\mathrm{e}}$ is even and smooth, we can assume that

$$
m_{\mathrm{e}}(\xi ; x) \sim m_{N}(\xi ; x) \equiv \sum_{n=0}^{N} \xi^{2 n} M_{n}(x) \quad \text { as } \xi \rightarrow 0
$$

where

$$
M_{n}(x)=\left.\frac{1}{(2 n) !} \frac{d^{2 n}}{d \xi^{2 n}} m_{\mathrm{e}}(\xi ; x)\right|_{\xi=0}=\left.\frac{1}{(2 n) !} \frac{d^{2 n}}{d t^{2 n}} m(t)\right|_{t=x}
$$

In particular, $M_{0}(x)=m(x)$.

In order to expand $I_{1}$, we use a standard technique, which is to use the Mellin transform $[2,5,10,11,13]$. We take the Mellin transform with respect to $r$; by definition,

$$
\mathscr{M}[f]=\tilde{f}(z)=\int_{0}^{\infty} f(r) r^{z-1} d r
$$

where $z$ is the (complex) transform variable. From relation (3.6), it follows that $\mathscr{M}\left\{I_{1}\right\}=\tilde{I}_{1}(z ; x)$ is analytic in the strip $0<\operatorname{Re} z<1$. To see this, note first that $I_{1}(r, x)=O\left(r^{-1}\right)$ as $r \rightarrow \infty$; this gives $\operatorname{Re} z<1$. Second, $I_{1}(r, x)=O(\log r)$ as $r \rightarrow 0$, since

$$
\int_{0}^{a} \frac{d \xi}{\sqrt{\xi^{2}+r^{2}}}=\log \left(\frac{a+\sqrt{a^{2}+r^{2}}}{r}\right)
$$

this gives $\operatorname{Re} z>0$.

Explicit calculation shows that

$$
\tilde{I}_{l}(z ; x)=B(z) C(z ; x)
$$

where

$$
\begin{aligned}
B(z) & =2 \int_{0}^{\infty} \frac{t^{z-1} d t}{\sqrt{1+t^{2}}}=\frac{1}{\sqrt{\pi}} \Gamma\left(\frac{1-z}{2}\right) \Gamma\left(\frac{z}{2}\right) \\
C(z ; x) & =\int_{0}^{a} m_{\mathrm{e}}(\xi ; x) \xi^{z-1} d \xi
\end{aligned}
$$

Later, we shall investigate the singularities of these functions.

The Mellin inversion formula gives

$$
I_{1}(r, x)=\frac{1}{2 \pi i} \int_{c-i \infty}^{c+i \infty} \tilde{I}_{1}(z ; x) r^{-z} d z
$$

with $0<c<1$. To obtain the expansion of $I_{1}$ in ascending powers of $r$, we move the inversion contour to the left, picking up residue contributions; this procedure will 
be justified in Section 3.1. We shall see that $\widetilde{I}_{1}(z ; x)$ has double poles at $z=-2 N$, $N=0,1,2, \ldots$ These will generate logarithmic terms because

$$
\begin{aligned}
r^{-z} & =r^{2 N} \exp \{-(z+2 N) \log r\} \\
& \simeq r^{2 N}\{1-(z+2 N) \log r\} \quad \text { near } z=-2 N ;
\end{aligned}
$$

here, we have used $a^{z} \sim 1+z \log a$ as $z \rightarrow 0$.

We know that $\Gamma(z)$ has simple poles at $z=-N, N=0,1,2, \ldots$; near $z=-N$ (see Equation (11) in Section 1.17 of [4])

$$
\Gamma(z) \simeq \frac{(-1)^{N}}{N !}\left\{\frac{1}{z+N}+\psi(N+1)\right\}
$$

where $\psi(z)=\left[\Gamma^{\prime}(z)\right] / \Gamma(z)$ is the psi function [1, page 258]. Hence $\Gamma(z / 2)$ has a similar approximation near $z=-2 N$. Also, a Taylor expansion gives

$\Gamma\left(\frac{1-z}{2}\right) \simeq \Gamma(N+1 / 2)\left\{1-\frac{1}{2}(z+2 N) \psi(N+1 / 2)\right\} \quad$ near $z=-2 N$.

Hence, we find that

$$
B(z) \simeq B_{N}\left\{\frac{1}{z+2 N}+\Psi_{N}\right\} \quad \text { near } z=-2 N,
$$

with

$$
B_{N}=\frac{2(-1)^{N} \Gamma(N+1 / 2)}{\sqrt{\pi} N !}=\frac{(-1)^{N}(2 N) !}{2^{2 N-1}(N !)^{2}}
$$

(using the duplication formula for $\Gamma[1$, Equation 6.1.18]) and

$$
\Psi_{N}=\frac{\psi(N+1)-\psi(N+1 / 2)}{2} .
$$

Thus, $B(z)$ has a simple pole at $z=-2 N, N=0,1,2, \ldots$ In particular, we have $B_{0}=2$ and $\Psi_{0}=\log 2$.

Next, we examine $C(z ; x)$. The definition (3.9) shows that $C(z ; x)$ is analytic for $\operatorname{Re} z>0$, but we must find its analytic continuation into $\operatorname{Re} z \leq 0$. A consequence of (3.7) is that (the analytic continuation of) $C$ also has simple poles at $z=-2 N$; we need an approximation similar to that found above for $B(z)$.

Subtracting $m_{N}$ from $m_{\mathrm{e}}$ in (3.9), we obtain

$$
C(z ; x)=\sum_{n=0}^{N} \frac{a^{z+2 n} M_{n}(x)}{z+2 n}+\int_{0}^{a}\left[m_{\mathrm{e}}(\xi ; x)-m_{N}(\xi ; x)\right] \xi^{z-1} d \xi .
$$

In this formula, the integral is analytic for $\operatorname{Re} z>-2 N-2$ whereas the series is meromorphic. Thus (3.13) exhibits the poles of $C(z ; x)$ and it enables us to 
approximate $C$ near these poles. Hence, as $a^{z+2 N} \simeq 1+(z+2 N) \log a$ when $z \simeq-2 N$, we obtain

where

$$
C(z ; x) \simeq \frac{M_{N}(x)}{z+2 N}+M_{N}(x) \log a+C_{N}(x) \quad \text { near } z=-2 N,
$$

$$
C_{N}(x)=\sum_{n=0}^{N-1} \frac{a^{2 n-2 N}}{2 n-2 N} M_{n}(x)+\int_{0}^{a} \frac{m_{\mathrm{e}}(\xi ; x)-m_{N}(\xi ; x)}{\xi^{2 N+1}} d \xi
$$

and the sum is absent if $N=0$. Thus $C(z ; x)$ has a simple pole at $z=-2 N$, $N=0,1,2, \ldots$.

We conclude that $B C r^{-z}$ has a double pole at $z=-2 N, N=0,1,2, \ldots$. Writing $z+2 N=w$, we see that

$$
B C r^{-z} \simeq r^{2 N} B_{N}(1-w \log r)\left(\frac{M_{N}}{w}+M_{N} \log a+C_{N}\right)\left(\frac{1}{w}+\Psi_{N}\right)
$$

near $w=0$, and so the residue at $z=-2 N$ is

$$
r^{2 N} B_{N}\left\{M_{N}\left(\Psi_{N}+\log \left(\frac{a}{r}\right)\right)+C_{N}\right\}
$$

Summing up,

$$
I_{1}(r, x)=\sum_{n=0}^{N} r^{2 n} B_{n}\left\{M_{n}\left(\Psi_{n}+\log \left(\frac{a}{r}\right)\right)+C_{n}\right\}+R_{N}(r, x)
$$

where

$$
R_{N}(r, x)=O\left(r^{2 N+2} \log r\right) \quad \text { as } r \rightarrow 0
$$

Combining this result with (3.1) and (3.3), we obtain the complete asymptotic expansion of $\phi$.

3.1. Justification of (3.15) and (3.16). To justify moving the contour, consider $\int_{\mathscr{C}} B C r^{-z} d z$, where $\mathscr{C}$ is the closed rectangle given by

$$
\mathscr{C}=\mathscr{C}_{0} \cup \mathscr{C}_{+} \cup \mathscr{C}_{-} \cup \mathscr{C}_{N}
$$

with positive (anti-clockwise) orientation. Put $z=\sigma+i \tau$. Then, $\mathscr{C}_{0}$ is $\sigma=c$, $|\tau|<Y$ with $0<c<1$ and $Y>0$; eventually, we shall let $Y \rightarrow \infty$. The left side of the rectangle is $\mathscr{C}_{N}$, defined by $\sigma=-2 N-\delta$ and $|\tau|<Y$ with $0<\delta<2$. Also, $\mathscr{C}_{ \pm}$is $\tau= \pm Y$ with $-2 N-\delta<\sigma<c$. Thus, $\mathscr{C}$ encloses the poles at $z=-2 n$, $n=0,1,2, \ldots, N$. 
From (3.13), we see that the analytic continuation of $C(z ; x)$ is bounded on $\mathscr{C}$. Also, as $\Gamma(z) \Gamma(1-z)=\pi / \sin (\pi z),(3.8)$ gives

$$
B(z)=\frac{\sqrt{\pi}}{\cos (\pi z / 2)} \frac{\Gamma(z / 2)}{\Gamma((z+1) / 2)} \sim \frac{\sqrt{2 \pi / z}}{\cos (\pi z / 2)}
$$

as $|z| \rightarrow \infty$, using [1, Equation 6.1.47]. Moreover, $2|\cos (\pi z / 2)| \sim e^{\pi|\tau| / 2}$ as $|\tau| \rightarrow \infty$. Hence, the integrals over $\mathscr{C}_{+}$and $\mathscr{C}_{-}$decay exponentially with increasing $Y$. Thus an application of the residue calculus gives (3.15) with

$$
\begin{aligned}
R_{N}(r, x) & =\lim _{Y \rightarrow \infty} \frac{1}{2 \pi i} \int_{\mathscr{C}_{N}} B C r^{-z} d z \\
& =\frac{r^{2 N+\delta}}{2 \pi} \int_{-\infty}^{\infty} B(i \tau-2 N-\delta) C(i \tau-2 N-\delta ; x) r^{-i \tau} d \tau .
\end{aligned}
$$

The integral is bounded (independently of $r$ ) and $\delta$ can be any number with $0<\delta<2$. For example, with $\delta=1$, we obtain $R_{N}(r, x)=O\left(r^{2 N+1}\right)$ as $r \rightarrow 0$. If we move the contour $\mathscr{C}_{N}$ further to the left, to $\mathscr{C}_{N+1}$, we obtain $R_{N}=\operatorname{term}_{N+1}+R_{N+1}$, where term is the $n$th term in (3.15). As $R_{N+1}=O\left(r^{2 N+3}\right)$, we see that $R_{N}$ is comparable to the first term neglected in (3.15), and so we obtain (3.16). For similar arguments, see [13, Section 5.1].

3.2. A special case. For a simple special case, take $N=0$ in (3.1), (3.3) and (3.15), giving

$$
\phi(r, x)=B_{0}\left\{M_{0}\left[\Psi_{0}+\log \left(\frac{a}{r}\right)\right]+C_{0}\right\}+c_{0}+O\left(r^{2} \log r\right) \quad \text { as } r \rightarrow 0 .
$$

Then, as $B_{0}=2, \Psi_{0}=\log 2$ and $M_{0}=m(x)$, we recover (2.1) in which

$$
f(x)=2 m(x) \log 2 a+2 C_{0}(x)+c_{0}(x) ;
$$

recall that $a=x-\alpha>0$. Now, from (3.14) and (3.5), we have

$$
\begin{aligned}
2 C_{0}(x)= & 2 \int_{0}^{a} \frac{m_{\mathrm{e}}(\xi ; x)-m(x)}{\xi} d \xi \\
= & \int_{0}^{a} \frac{m(x+\xi)-m(x)}{\xi} d \xi+\int_{0}^{a} \frac{m(x-\xi)-m(x)}{\xi} d \xi \\
= & \int_{x}^{x+a} \frac{m(t)-m(x)}{t-x} d t+\int_{x-a}^{x} \frac{m(t)-m(x)}{x-t} d t \\
= & {[m(x+a)+m(x-a)-2 m(x)] \log a+\int_{x-a}^{x} m^{\prime}(t) \log (x-t) d t } \\
& -\int_{x}^{x+a} m^{\prime}(t) \log (t-x) d t \\
= & {[m(x+a)+m(\alpha)-2 m(x)] \log 2 a+\int_{\alpha}^{x+a} m^{\prime}(t) L(x-t) d t, }
\end{aligned}
$$


as $x-a=\alpha ; L(x)$ is defined by (2.2). Also, (3.4) gives

$$
\begin{aligned}
c_{0}(x) & =\int_{2 x-\alpha}^{\beta} \frac{m(t) d t}{t-x} \\
& =m(\beta) \log 2(\beta-x)-m(x+a) \log 2 a+\int_{x+a}^{\beta} m^{\prime}(t) L(x-t) d t .
\end{aligned}
$$

When (3.19) and (3.20) are substituted in (3.17), we recover Goldstein's formula for $f,(2.4)$. Notice that if $m$ is not differentiable at $x$, then one can use $(3.18)$ for $C_{0}(x)$.

\section{End-point behaviour}

We have shown in Section 3 how to approximate $\phi(r, x)$ when $\alpha<x<\beta$. If $x<\alpha$ or $x>\beta$, the situation is simpler, because one merely uses the Maclaurin expansion (3.2) with $t=r /\left(x-x_{1}\right)$ in (1.1); the result is an expansion in powers of $r^{2}$.

The situation when $x=\alpha$ or $x=\beta$ is more interesting. Suppose that $x=\alpha$. Then (1.1) becomes

$$
\phi(r, \alpha)=\int_{0}^{\ell} \frac{m(\alpha+\xi)}{\sqrt{\xi^{2}+r^{2}}} d \xi,
$$

where $\ell=\beta-\alpha$. The behaviour of $\phi(r, \alpha)$ for small $r$ depends on the behaviour of $m(x)$ as $x \rightarrow \alpha+$. Let us assume that

$$
m(\alpha+\xi) \sim \sum_{n \geq 0} A_{n} \xi^{\kappa_{n}} \quad \text { as } \xi \rightarrow 0+,
$$

where $-1<\kappa_{0}<\kappa_{1}<\cdots$; the quantities $\kappa_{n}$ and $A_{n}$ are constants. The asymptotic form (4.1) allows for possible singular behaviour at the end-point, $x=\alpha$; logarithmic terms could also be included. (A similar analysis could be carried out at points $x$ with $\alpha<x<\beta$ where $m$ or one of its derivatives is discontinuous.)

Next, we calculate the Mellin transform of $\phi(r, \alpha)$, denoted by $\widetilde{\phi}(z, \alpha)$. This function is analytic in the strip $\max \left(0,-\kappa_{0}\right)<\operatorname{Re} z<1$, and is given by

$$
\widetilde{\phi}(z, \alpha)=B(z) C_{\alpha}(z)
$$

where $B(z)$ is defined by (3.8) and

$$
C_{\alpha}(z)=\frac{1}{2} \int_{0}^{\ell} m(\alpha+\xi) \xi^{z-1} d \xi .
$$

Proceeding as before, we move the inversion contour to the left. The first pole encountered is a simple pole at $z=-\kappa_{0}$ if $-1<\kappa_{0}<0$ but it is a simple pole at 
$z=0$ if $\kappa_{0}>0$. If $\kappa_{0}=0$, there is a double pole at $z=0$. In any of these cases, we can calculate the asymptotic expansion of $\phi(r, x)$ for small $r$ by computing residues. For example, suppose that $\kappa_{0}=0$ so that $m(\alpha+\xi) \sim A_{0}=m(\alpha)$ as $\xi \rightarrow 0+$. Then using (3.12) (with $N=0$ ) and

$$
2 C_{\alpha}(z) \simeq \frac{m(\alpha)}{z}+X_{0} \quad \text { near } z=0,
$$

where

$X_{0}=m(\alpha) \log \ell+\int_{0}^{\ell} \frac{m(\alpha+\xi)-m(\alpha)}{\xi} d \xi=m(\beta) \log \ell-\int_{0}^{\ell} m^{\prime}(\alpha+\xi) \log \xi d \xi$,

we find that

$\phi(r, \alpha) \sim-m(\alpha) \log \left(\frac{r}{2}\right)+m(\beta) \log (\beta-\alpha)-\int_{\alpha}^{\beta} m^{\prime}(\xi) \log (\xi-\alpha) d \xi$

as $r \rightarrow 0$; this should be compared with (2.3).

The result (4.5) holds when the field point at $(x, r)=(\alpha, r)$ approaches the point $(\alpha, 0)$ at one end of the line of sources in a direction perpendicular to the line. More generally, other approach directions could be selected. Thus, introduce spherical polar coordinates $(R, \theta)$, with

$$
x=\alpha-R \cos \theta, \text { and } r=R \sin \theta,
$$

where $R>0$ and $0 \leq \theta<\pi ; \theta$ is the angle of approach. Then, with a slight abuse of notation, (1.1) gives

$$
\phi(R, \theta)=\int_{\alpha}^{\beta} \frac{m\left(x_{1}\right) d x_{1}}{\sqrt{\left(\alpha-x_{1}-R \cos \theta\right)^{2}+R^{2} \sin ^{2} \theta}}=\int_{0}^{\ell} \frac{m(\alpha+\xi) d \xi}{\sqrt{\xi^{2}+2 \xi R \cos \theta+R^{2}}}
$$

with $\ell=\beta-\alpha$. Now, calculate the Mellin transform of $\phi(R, \theta)$ with respect to $R$,

$$
\widetilde{\phi}(z, \theta)=\int_{0}^{\infty} R^{z-1} \phi(R, \theta) d R=2 C_{\alpha}(z) \int_{0}^{\infty} \frac{t^{z-1} d t}{\sqrt{1+2 r \cos \theta+t^{2}}},
$$

with $C_{\alpha}$ defined by (4.2). As before, the function $\widetilde{\phi}(z, \theta)$ is analytic in the strip $\max \left(0,-\kappa_{0}\right)<\operatorname{Re} z<1$; see (4.1). The remaining integral in (4.6) can be evaluated [7, Equation $8.714(2)]$, giving

$$
\tilde{\phi}(z, \theta)=\frac{2 \pi}{\sin \pi z} C_{\alpha}(z) P_{-z}(\cos \theta), \quad \max \left(0,-\kappa_{0}\right)<\operatorname{Re} z<1,
$$

where $P_{v}(x)$ is a Legendre function. We can now proceed as before, moving the inversion contour to the left. 
Let us assume that $\kappa_{0}=0$ so that $\tilde{\phi}(z, \theta)$ has a double pole at $z=0$. Then, near $z=0,(4.7)$ gives

$$
\widetilde{\phi}(z, \theta) R^{-z} \simeq \frac{1}{z}\left(\frac{m(\alpha)}{z}+X_{0}\right)\left[1-2 z \log \left(\cos \left(\frac{\theta}{2}\right)\right)\right](1-z \log R),
$$

where we have used (3.10) (with $N=0$ ), (4.3) and [1, Equation 8.6.20]. Hence, computing the residue at $z=0$, we obtain

$$
\phi(R, \theta) \sim-m(\alpha) \log \left[R \cos ^{2}\left(\frac{\theta}{2}\right)\right]+X_{0} \quad \text { as } R \rightarrow 0, \text { with } 0 \leq \theta<\pi,
$$

where the constant $X_{0}$ is given by (4.4). Notice that

$$
\left.\frac{\partial}{\partial \nu}\left[R^{\nu} P_{\nu}(\cos \theta)\right]\right|_{\nu=0}=\log \left[R \cos ^{2}\left(\frac{\theta}{2}\right)\right]
$$

is an axisymmetric harmonic function. Notice also that $(4.8)$ reduces to $(4.5)$ when $\theta=\pi / 2$.

We conclude by noting that the Mellin-transform technique is applicable to other related problems. For example, it can be used to estimate

$$
\int_{\alpha}^{\beta} \frac{m\left(x_{1}\right) d x_{1}}{\left[\left(x-x_{1}\right)^{2}+r^{2}\right]^{\mu}}
$$

where $\mu$ is a parameter; the case $\mu=3 / 2$ is of some interest [15].

\section{Acknowledgements}

The paper has benefitted from the comments of two referees. The calculation leading to (4.8) was made after a stimulating conversation with Xavier Claeys, a PhD student at INRIA Rocquencourt.

\section{References}

[1] M. Abramowitz and I. A. Stegun (eds.), Handbook of mathematical functions (Dover, New York, 1965).

[2] N. Bleistein and R. A. Handelsman, Asymptotic expansions of integrals (Holt, Rinehart and Winston, New York, 1975).

[3] E. Chadwick, "A slender-body theory in Oseen flow", Proc. Roy. Soc. A 458 (2002) 2007-2016.

[4] A. Erdélyi, W. Magnus, F. Oberhettinger and F. G. Tricomi, Higher transcendental functions vol. I (McGraw-Hill, New York, 1953). 
[5] G. Fikioris, "Integral evaluation using the Mellin transform and generalized hypergeometric functions: Tutorial and applications to antenna problems", IEEE Trans. Antennas \& Propagation 54 (2006) 3895-3907.

[6] S. Goldstein, Lectures on fluid mechanics (Interscience, London, 1960).

[7] I. S. Gradshteyn and I. M. Ryzhik, Table of integrals, series, and products (Academic Press, London, 1980).

[8] R. A. Handelsman and J. B. Keller, "Axially symmetric potential flow around a slender body", $J$. Fluid Mech. 28 (1967) 131-147.

[9] R. A. Handelsman and J. B. Keller, "The electrostatic field around a slender conducting body of revolution", SIAM J. Appl. Math. 15 (1967) 824-841.

[10] P. A. Martin, "End-point behaviour of solutions to hypersingular integral equations", Proc. Roy. Soc. A 432 (1991) 301-320.

[11] P. A. Martin, "Asymptotic approximations for functions defined by series, with some applications to the theory of guided waves", IMA J. Appl. Math. 54 (1995) 139-157.

[12] J. P. Moran, "Line source distributions and slender-body theory",J. Fluid Mech. 17 (1963) 285-304.

[13] R. B. Paris and D. Kaminski, Asymptotics and Mellin-Barnes integrals (Cambridge University Press, Cambridge, 2001).

[14] A. G. Petrov, "Asymptotic expansions for axially symmetric cavities", Euro. J. Appl. Math. 16 (2005) 319-334.

[15] A. Sellier, "A general and formal slender-body theory in the non-lifting case", Proc. Roy. Soc. A 453 (1997) 1733-1751.

[16] E. O. Tuck, "Some methods for flows past blunt slender bodies", J. Fluid Mech. 18 (1964) 619-635.

[17] E. O. Tuck, "Analytic aspects of slender body theory", in Wave asymptotics (Manchester, 1990), (Cambridge University Press, Cambridge, 1992) 184-201. 